\title{
Stability and Optimal Control of a Delayed HIV Model
}

\author{
Diana Rocha ${ }^{a}$, Cristiana J. Silva ${ }^{a}$ and Delfim F. M. Torres ${ }^{a *}$
}

\begin{abstract}
We propose and investigate a delayed model that studies the relationship between HIV and the immune system during the natural course of infection and in the context of antiviral treatment regimes. Sufficient criteria for local asymptotic stability of the infected and viral free equilibria are given. An optimal control problem with time delays both in state variables (incubation delay) and control (pharmacological delay) is then formulated and analyzed, where the objective consists to find the optimal treatment strategy that maximizes the number of uninfected $C D 4^{+} \mathrm{T}$ cells as well as CTL immune response cells, keeping the drug therapy as low as possible.
\end{abstract}

Keywords: HIV modelling; incubation and pharmacological time delays; stability; optimal control.

\section{Introduction}

The study of mathematical models for human immunodeficiency virus (HIV) infection is a subject of strong current interest, both at population and cell levels (see, e.g., [12, 17, 18] and references cited therein). Based on the model of [5], in this work we analyze a mathematical model that studies the relationship between HIV and the immune system during the natural course of infection and in the context of antiviral treatment regimes. The model considers three variables: uninfected $C D 4^{+} \mathrm{T}$ cells, denoted by $x$; infected $C D 4^{+} T$ cells, denoted by $y$; and $C T L$ effectors (immune response cells), denoted by $z$. According to [1], the viral load is assumed to be proportional to the level of infected cells. Uninfected $C D 4^{+} \mathrm{T}$ cells are produced at a rate $\lambda$, die at a rate $d$, and become infected at a rate $\beta$. Infected cells decay at a rate $a$ and are killed by CTL effectors at a rate $p$. Proliferation of the CTL population is given by cxyz and is proportional to both virus load $(y)$ and the number of uninfected $C D 4^{+} \mathrm{T}$ cells $(x)$. CTL effectors die at a rate $h$. Mathematically, the model [5] is described by

$$
\left\{\begin{array}{l}
\dot{x}=\lambda-d x-\beta x y \\
\dot{y}=\beta x y-a y-p y z \\
\dot{z}=c x y z-h z .
\end{array}\right.
$$

Time delays play an important role in the dynamics of HIV infection: see, e.g., $[13,14]$ and references therein. For this reason, in this work we introduce a discrete time-delay into the model (1), which represents the incubation period, that is, the time between the new infection of a $C D 4^{+} \mathrm{T}$ cell and the time it becomes infectious (Section 2). We prove local asymptotic stability of the viral free and infected equilibriums, for any time delay (Section 3).

Optimal control theory has been applied with success to epidemiology HIV models: see, e.g., [5, 8] and references therein. However, epidemiology optimal control problems with delays in both state and control variables are a rarity. For one such optimal control problem, of a tuberculosis model, we refer the reader to the recent work of Silva, Maurer and Torres [16]. Here, we propose and solve a HIV optimal control problem, with delays in both state and control variables, where the objective is to find the optimal treatment strategy that maximizes the number of $C D 4^{+} \mathrm{T}$ cells, as well as $\mathrm{CTL}$ immune response cells, keeping the drug/chemotherapy strength, which depends on a pharmacological delay, as low as possible (Section 4). The stability and optimal control results of Sections 3 and 4 are then illustrated through numerical simulations in Section 5 . We end with Section 6 of conclusions and future work.

\footnotetext{
a Center for Research and Development in Mathematics and Applications (CIDMA), Department of Mathematics, University of Aveiro, $3810-193$ Aveiro, Portugal

* Correspondence to: Department of Mathematics, University of Aveiro, 3810-193 Aveiro, Portugal. Email: delfim@ua.pt
} 


\section{Model with time delay $\tau$}

In epidemiological literature, a latent or incubation period is often modeled by incorporating it as a delay effect [10]. We consider the following delayed model, where $\tau>0$ represents the incubation period:

$$
\left\{\begin{array}{l}
\dot{x}(t)=\lambda-d x(t)-\beta x(t) y(t), \\
\dot{y}(t)=\beta x(t-\tau) y(t-\tau)-a y(t)-p y(t) z(t), \\
\dot{z}(t)=c x(t) y(t) z(t)-h z(t) .
\end{array}\right.
$$

The initial conditions for system (2) are

$$
x(\theta)=\varphi_{1}(\theta), \quad y(\theta)=\varphi_{2}(\theta), \quad z(\theta)=\varphi_{3}(\theta),
$$

$-\tau \leq \theta \leq 0$, where $\varphi=\left(\varphi_{1}, \varphi_{2}, \varphi_{3}\right)^{T} \in C$ with $C$ the Banach space $C\left([-\tau, 0], \mathbb{R}^{3}\right)$ of continuous functions mapping the interval $[-\tau, 0]$ into $\mathbb{R}^{3}$. The usual local existence, uniqueness and continuation results apply [7, 11]. Therefore, there exists a unique solution $(x(t), y(t), z(t))$ of (2) with initial conditions (3), for all time $t \geq 0$. From biological meaning, we further assume the initial functions to be non-negative, that is,

$$
\varphi(\theta) \geq 0 \quad \text { for } \quad \theta \in[-\tau, 0], \quad i=1, \ldots, 3 .
$$

From [19, Theorem 2.1], it follows that all solutions of (2) satisfying (3) with (4) are bounded for all time $t \geq 0$, which ensures not only local existence but also the existence of a solution for all time $t \geq 0$.

It is easy to see that system (2) has an infection-free equilibrium

$$
E_{0}=\left(\frac{\lambda}{d}, 0,0\right)
$$

which is the only biologically meaningful equilibrium if $\beta<\frac{d a}{\lambda}$. Let $\beta>\frac{d a}{\lambda}$ and assume that $\lambda c-\beta h>0$. If $\beta<\frac{\text { acd }}{\lambda c-\beta h}$, then system (2) has a unique infected equilibrium $E_{1}$ given by

$$
E_{1}=\left(\frac{a}{\beta}, \frac{\lambda \beta-d a}{\beta a}, 0\right) .
$$

Assume that $\lambda c-\beta h>0$. Whenever $\beta>\frac{a c d}{\lambda c-\beta h}$, the unique infected equilibrium of system (2) is given by the CTL equilibrium

$$
E_{2}=\left(\frac{\lambda c-\beta h}{c d}, \frac{d h}{\lambda c-\beta h}, \frac{\beta(\lambda c-\beta h)}{c d p}-\frac{a}{p}\right) .
$$

\section{Local asymptotic stability}

Consider the following coordinate transformation:

$$
X(t)=x(t)-\bar{x}, \quad Y(t)=y(t)-\bar{y}, \quad Z(t)=z(t)-\bar{z},
$$

where $(\bar{x}, \bar{y}, \bar{z})$ denotes any equilibrium of $(2): E_{0}, E_{1}$ or $E_{2}$. The linearized system of (2) is of form

$$
\left\{\begin{array}{l}
\dot{X}(t)=(-d-\beta \bar{y}) X(t)-\beta \bar{x} Y(t), \\
\dot{Y}(t)=(-a-p \bar{z}) Y(t)+\beta \bar{y} X(t-\tau)+\beta \bar{x} Y(t-\tau)-p \bar{y} Z(t), \\
\dot{Z}(t)=c \bar{y} \bar{z} X(t)+c \bar{x} \bar{z} Y(t)+(c \bar{x} \bar{y}-h) Z(t) .
\end{array}\right.
$$

We can express system (8) in matrix notation as follows:

$$
\frac{d}{d t}\left(\begin{array}{c}
X(t) \\
Y(t) \\
Z(t)
\end{array}\right)=A_{1}\left(\begin{array}{c}
X(t) \\
Y(t) \\
Z(t)
\end{array}\right)+A_{2}\left(\begin{array}{c}
X(t-\tau) \\
Y(t-\tau) \\
Z(t-\tau)
\end{array}\right)
$$

where $A_{1}$ and $A_{2}$ are the $3 \times 3$ matrices given by

$$
A_{1}=\left(\begin{array}{ccc}
-d-\beta \bar{y} & -\beta \bar{x} & 0 \\
0 & -a-p \bar{z} & -p \bar{y} \\
c \bar{y} \bar{z} & c \bar{x} \bar{z} & c \bar{x} \bar{y}-h
\end{array}\right)
$$

and

$$
A_{2}=\left(\begin{array}{ccc}
0 & 0 & 0 \\
\beta \bar{y} & \beta \bar{x} & 0 \\
0 & 0 & 0
\end{array}\right)
$$




\subsection{Local stability of the infection-free equilibrium $E_{0}$ for any time delay $\tau$}

The characteristic equation of system (2) is given by

$$
\Delta\left(\lambda_{1}\right)=\operatorname{det}\left(\lambda_{1} / d-A_{1}-e^{-\lambda_{1} \tau} A_{2}\right)=0,
$$

where $/ d$ denotes the identity matrix of dimension 3. The following result holds.

Theorem 1 (i) If $\beta \lambda-a d<0$, then the infection-free equilibrium $E_{0}$ (5) is locally asymptotically stable for any time delay $\tau \geq 0$. (ii) If $\beta \lambda-a d>0$, then $E_{0}$ (5) is unstable for any time-delay $\tau \geq 0$. (iii) If $\beta \lambda-a d=0$, then a critical case occurs.

Proof: (i) The characteristic equation (9) associated to the infection-free equilibrium is given by

$$
\frac{\left(d+\lambda_{1}\right)\left(h+\lambda_{1}\right)\left(a d+d \lambda_{1}-\beta \lambda e^{-\lambda_{1} \tau}\right)}{d}=0 .
$$

Assume that $\tau=0$. In this case, the equation (10) becomes

$$
\frac{\left(d+\lambda_{1}\right)\left(h+\lambda_{1}\right)\left(a d+d \lambda_{1}-\beta \lambda e^{-\lambda_{1} \tau}\right)}{d}=0 .
$$

The roots of (11) are $-d,-h$ and $\frac{\beta \lambda-d a}{d}$, which have negative real part when $\beta \lambda-d a<0$. Suppose that $\tau>0$. To prove the stability of $E_{0}$ we use Rouché's theorem. Thus, we need to prove that the roots of the characteristic equation (10) cannot intersect the imaginary axis, i.e., the characteristic equation cannot have pure imaginary roots. Suppose the contrary, i.e., that there exists a nonzero $w$ such that $b=w i$ is a solution of (10). Equation (10) has two real negative solutions $-d$ and $-h$. Therefore, in what follows we just consider the term $\frac{a d+d \lambda_{1}-\beta \lambda e^{-\lambda_{1} \tau}}{d}$. The complex $b=w i$ is a root of $(10)$ if $\frac{a d+d w i-\beta \lambda e^{-w i \tau}}{d}=0$. By using Euler's formula $\exp ^{-i w \tau}=\cos (w \tau)-i \sin (w \tau)$, and by separating real and imaginary parts, we have

$$
\left\{\begin{array}{l}
d a=\beta \lambda \cos (w \tau) \\
d w=-\beta \lambda \sin (w \tau) .
\end{array}\right.
$$

Adding up the squares of both equations, we obtain that

$$
d^{2} w^{2}+d^{2} a^{2}-\lambda^{2} \beta^{2}=0,
$$

that is,

$$
w^{2}=\frac{\lambda^{2} \beta^{2}-d^{2} a^{2}}{d^{2}}
$$

If $\beta \lambda-d a<0$, then $w^{2}<0$, which is a contradiction. We just proved that the characteristic equation (10) cannot have pure imaginary roots and the infection-free equilibrium $E_{0}$ is locally asymptotically stable for any strictly positive time delay.

(ii) Suppose now that $\beta \lambda-d a>0$. We know that the characteristic equation (10) has two real negative roots: $\lambda_{1}=-d$ and $\lambda_{1}=-h$. Thus, we must check if the remaining roots of function $f\left(\lambda_{1}\right):=a+\lambda_{1}-\frac{\beta \lambda_{e}{ }^{-\lambda_{1} \tau}}{d}$ have negative real parts. It is easy to see that $f(0)=a-\frac{\beta \lambda}{d}<0$, since we are assuming $\beta \lambda-d a>0$. On the other hand, $\lim _{\lambda_{1} \rightarrow+\infty} f\left(\lambda_{1}\right)=+\infty$. Therefore, by continuity of $f\left(\lambda_{1}\right)$, there is at least one positive root of the characteristic equation (10). Hence, we conclude that $E_{0}$ is unstable.

(iii) Finally, we analyze the case $\beta \lambda-d a=0$. In this situation, the characteristic equation (10) becomes

$$
\left(d+\lambda_{1}\right)\left(h+\lambda_{1}\right)\left(\lambda_{1}+a-\exp ^{-\lambda_{1} \tau} a\right)=0 .
$$

To prove the stability we need to check again if all the roots of the above equation have negative real parts. Notice that $\lambda_{1}=0$, $\lambda_{1}=-d$ and $\lambda_{1}=-h$ are solutions of this equation, so we just need to prove that the remaining roots cannot have nonnegative real parts. Assuming that $\lambda_{1}=u+$ wi with $u \geq 0$ is a solution of the above equation, then

$$
u+w i+a-\exp ^{-(u+w i) \tau} a=0 .
$$

By using Euler formula and separating the real and imaginary parts, we get

$$
\left\{\begin{array}{l}
u+a=e^{-u \tau} a \cos (w \tau) \\
w=-e^{-u \tau} a \sin (w \tau) .
\end{array}\right.
$$

By adding up the squares of both equations, and using the fundamental trigonometric formula, we obtain

$$
(u+a)^{2}+w^{2}=\exp (-2 u \tau) a^{2} \leq a^{2},
$$

which is a contradiction. This proves that 0 is the unique root of (12) that does not have negative real part. 


\subsection{Local stability of the infected equilibrium $E_{1}$ for any time delay $\tau$}

We now study the local stability of the the infected equilibrium $E_{1}(6)$ for any incubation period $\tau$.

Theorem 2 Let $\beta \lambda-d a>0$ and assume that $\lambda c-\beta h>0$. (i) If $\beta(\lambda c-\beta h)-a c d<0$, then the infected equilibrium $E_{1}$ is locally asymptotically stable for any time delay $\tau \geq 0$. (ii) If $\beta(\lambda c-\beta h)-$ acd $>0$, then $E_{1}$ is unstable for any time delay $\tau \geq 0$.

Proof: Let $\beta \lambda-d a>0$ and $\lambda c-\beta h>0$. The characteristic equation (9) at $E_{1}=\left(\frac{a}{\beta}, \frac{\lambda \beta-d a}{\beta a}, 0\right)$ is given by

$$
\frac{\left(\lambda_{1} \beta^{2}-\beta \lambda c+\beta^{2} h+a c d\right)\left(-\lambda_{1}^{2} a-\lambda_{1} a^{2}+\lambda_{1} a^{2} e^{-\lambda_{1} \tau}-\lambda \beta \lambda_{1}-\lambda \beta a+e^{-\lambda_{1} \tau} a^{2} d\right)}{a \beta^{2}}=0 .
$$

Note that $\lambda_{1}=\frac{\beta \lambda c-\beta^{2} h-a c d}{\beta^{2}}$ is a solution of (13). (i) If $\beta(\lambda c-\beta h)-$ acd $<0$, then $\lambda_{1}=\frac{\beta \lambda c-\beta^{2} h-a c d}{\beta^{2}}$ is a real negative root of the characteristic equation (13) and we just need to analyze the equation

$$
\frac{\lambda_{1}^{2} a+\lambda_{1}\left(a^{2}+\lambda \beta\right)+\lambda \beta a-\left(\lambda_{1}+d\right) a^{2} e^{-\lambda_{1} \tau}}{a \beta^{2}}=0
$$

Consider $\tau=0$. From equation (14), we have

$$
\frac{\lambda_{1}^{2} a+\lambda \beta \lambda_{1}+\lambda \beta a-a^{2} d}{a \beta^{2}}=0 .
$$

Since $\beta \lambda-d a>0$, it follows that $\frac{1}{\beta^{2}}>0, \frac{\lambda}{a \beta}>0$ and $\frac{\lambda \beta a-a^{2} d}{a \beta^{2}}=\frac{\lambda \beta-a d}{\beta^{2}}>0$. Therefore, from the Routh-Hurwitz criterion, it follows that all roots of (15) have negative real part. Hence, $E_{1}$ is locally asymptotically stable for $\tau=0$. Let $\tau>0$. Suppose that (14) has pure imaginary roots \pm wi. By replacing $\lambda_{1}$ in (14) by $w i$, and separate the real and imaginary parts, we obtain

$$
\left\{\begin{array}{l}
-w^{2} a+\lambda \beta a=a^{2} d \cos (w \tau)+w a^{2} \sin (w \tau) \\
w a^{2}+\lambda \beta w=w a^{2} \cos (w \tau)-a^{2} d \sin (w \tau) .
\end{array}\right.
$$

By adding up the squares of both equations, and using the fundamental trigonometric formula, we obtain that

$$
a^{2} w^{4}+w^{2} \lambda^{2} \beta^{2}=a^{2}\left(a^{2} d^{2}-\lambda^{2} \beta^{2}\right),
$$

which is a contradiction since $\beta \lambda-d a>0$. Therefore, $a^{2} d^{2}-\lambda^{2} \beta^{2}<0$ and equation (14) does not have pure imaginary roots. This implies that $E_{1}$ is locally asymptotically stable for any time delay $\tau>0$. (ii) If $\beta>\frac{\text { acd }}{\lambda c-\beta h}$, then the characteristic equation (13) has a positive root and consequently the equilibrium $E_{1}$ is unstable for any time delay $\tau \geq 0$.

\subsection{Local stability of the CTL equilibrium $E_{2}$}

The analysis of the local stability of the CTL equilibrium $E_{2}$ is more complex. Under some assumptions, the situation is clear for $\tau=0$ : the infected equilibrium $E_{2}$ is locally asymptotically stable (see Theorem 3 ). However, for $\tau>0$, the characteristic polynomial has pure imaginary roots and we are not able to conclude anything about the stability for an arbitrary $\tau>0$ (see Remark 4). It is, however, possible to prove stability in some concrete situations of biological significance (see Remark 5 ).

Theorem 3 Assume that $\lambda c-\beta h>0$. If $\beta(\lambda c-\beta h)-a c d>0$, then the infected equilibrium $E_{2}$ is locally asymptotically stable for $\tau=0$

Proof: Let $\beta(\lambda c-\beta h)-a c d>0$. The characteristic equation (9) at $E_{2}(7)$ is given by

$$
\lambda_{1}^{3}+A \lambda_{1}^{2}+B \lambda_{1}+C+\left(-\frac{\lambda_{1} \beta\left(\lambda_{1}(c \lambda-\beta h)+d(c \lambda-\beta h)\right)}{c d}\right) \exp \left(-\lambda_{1} \tau\right)=0,
$$

where

$$
A=\frac{\lambda^{2} c^{2} \beta+d^{2} \lambda c^{2}-2 \lambda c \beta^{2} h+\beta^{3} h^{2}}{(\lambda c-\beta h) c d}, \quad B=\frac{c \lambda d \beta+c \lambda \beta h-c h a d-\beta^{2} h^{2}}{c d} \text { and } C=-\frac{\left(-\beta \lambda c+\beta^{2} h+a c d\right) h}{c} .
$$

If $\tau=0$, then the characteristic equation (16) is $\lambda_{1}^{3}+D \lambda_{1}^{2}+E \lambda_{1}+F=0$ with $D=\frac{d \lambda c}{\lambda c-\beta h}>0, E=\frac{\left(\beta \lambda c-a c d+d \beta^{2}-\beta^{2} h\right) h}{c d}>0$, $F=\frac{\left(\beta \lambda c-\beta^{2} h-a c d\right) h}{c}>0$ and $D E-F=\frac{h \beta\left(c \lambda d \beta+h\left(c \lambda \beta-c a d-\beta^{2} h\right)\right)}{(\lambda c-\beta h) c}>0$, whenever $\beta(\lambda c-\beta h)-\operatorname{acd}>0$. 
Remark 4 Let $\tau>0$. Suppose that (16) has pure imaginary roots \pm wi. Replacing $\lambda_{1}$ in (16) by wi, and separating the real and imaginary parts, we obtain that

$$
\left\{\begin{array}{l}
G w^{2}+H=J w^{2}+K w \\
L w^{3}+M w=N w^{2}+P w
\end{array}\right.
$$

where

$$
\begin{aligned}
G & =-\beta^{3} h^{2}-\beta c^{2} \lambda^{2}-c^{2} d^{2} \lambda+2 \beta^{2} c h \lambda, \\
H & =d \lambda^{2} c^{2} h \beta+h^{2} \beta d^{2} a c-d^{2} \lambda c^{2} h a-2 d \lambda c \beta^{2} h^{2}+h^{3} \beta^{3} d, \\
J & =\beta\left(\beta^{2} h^{2} \cos (w \tau)+\lambda^{2} c^{2} \cos (w \tau)-2 \lambda c \beta h \cos (w \tau)\right) \\
K & =\beta\left(-d \lambda^{2} c^{2} \sin (w \tau)+2 d \lambda c \beta h \sin (w \tau)-\beta^{2} h^{2} d \sin (w \tau)\right), \\
L & =-\lambda c^{2} d+\beta h c d, \\
M & =\beta^{3} h^{3}-\lambda c^{2} h a d-d \lambda c \beta^{2} h+\beta h^{2} a c d-2 \lambda c \beta^{2} h^{2}+d \lambda^{2} c^{2} \beta+\lambda^{2} c^{2} h \beta, \\
N & =-\beta\left(\sin (w \tau) c^{2} \lambda^{2}-2 \sin (w \tau) \beta c h \lambda+\sin (w \tau) \beta^{2} h^{2}\right) \\
P & =-\beta\left(\cos (w \tau) \beta^{2} d h^{2}-2 \cos (w \tau) \beta c d h \lambda+\cos (w \tau) c^{2} d \lambda^{2}\right) .
\end{aligned}
$$

By adding up the squares of both equations, and using the fundamental trigonometric formula, we obtain that

$$
Q w^{6}+R w^{4}+S w^{2}+T=0,
$$

where

$$
\begin{aligned}
Q & =\lambda^{2} c^{4} d^{2}+\beta^{2} h^{2} c^{2} d^{2}-2 \lambda c^{3} d^{2} \beta h, \\
R & =6 \lambda^{2} c^{3} d \beta^{2} h^{2}+2 \lambda^{2} c^{4} d^{2} h a+d^{4} \lambda^{2} c^{4}+2 \beta^{4} h^{4} c d-2 \lambda^{3} c^{4} d c h \beta+2 \beta^{2} h^{3} a c^{2} d^{2}-6 \beta^{3} h^{3} \lambda c^{2} d-4 \lambda c^{3} d^{2} \beta h^{2} a, \\
S & =-2 \beta^{6} h^{5} d-\beta^{6} d^{2} h^{4}-2 d^{3} \lambda^{3} c^{4} h \beta-2 h^{3} \beta^{3} d^{3} \lambda c^{2}-2 \beta^{4} h^{4} d^{2} a c+2 d^{4} \lambda^{2} c^{4} h a \\
& +4 d^{3} \lambda^{2} c^{3} \beta^{2} h^{2}+4 \beta^{5} h^{3} d^{2} \lambda c-6 \beta^{3} h^{4} \lambda c^{2} a d-2 \lambda^{2} c^{3} h^{2} a d^{2} \beta^{2}-2 \lambda c^{3} h^{3} a^{2} d^{2} \beta \\
& +6 \lambda^{2} c^{3} h^{3} a d \beta^{2}-2 \lambda^{3} c^{4} h^{2} a d \beta+4 d^{2} \lambda c^{2} \beta^{3} h^{3} a+\beta^{6} h^{6}-4 \beta^{5} h^{5} \lambda c+6 \beta^{4} h^{4} \lambda^{2} c^{2} \\
& -4 \lambda^{3} c^{3} \beta^{3} h^{3}+\lambda^{4} c^{4} h^{2} \beta^{2}-2 h^{2} \beta d^{4} a c^{3} \lambda+6 \beta^{5} h^{4} d \lambda c+2 \beta^{4} h^{5} a c d-6 \beta^{4} h^{3} d \lambda^{2} c^{2} \\
& +\lambda^{2} c^{4} h^{2} a^{2} d^{2}-5 d^{2} \lambda^{2} c^{2} \beta^{4} h^{2}+2 d^{2} \lambda^{3} c^{3} \beta^{3} h+2 d \lambda^{3} c^{3} \beta^{3} h^{2}+\beta^{2} h^{4} a^{2} c^{2} d^{2} \\
T & =-4 d^{2} \lambda^{3} c^{3} \beta^{3} h^{3}-4 d^{2} \lambda c \beta^{5} h^{5}+d^{2} \lambda^{4} c^{4} h^{2} \beta^{2}-2 h^{3} \beta d^{4} a^{2} c^{3} \lambda-6 h^{4} \beta^{3} d^{3} a c^{2} \lambda+h^{6} \beta^{6} d^{2} \\
& +h^{4} \beta^{2} d^{4} a^{2} c^{2}+2 h^{5} \beta^{4} d^{3} a c+6 h^{3} \beta^{2} d^{3} a c^{3} \lambda^{2}-2 d^{3} \lambda^{3} c^{4} h^{2} a \beta+d^{4} \lambda^{2} c^{4} h^{2} a^{2}+6 d^{2} \lambda^{2} c^{2} \beta^{4} h^{4} .
\end{aligned}
$$

This equation admits at least two pure imaginary roots. Indeed, let $\lambda=1, d=\frac{1}{10}, \beta=\frac{1}{2}, a=\frac{1}{5}, p=1, c=\frac{1}{10}$ and $h=\frac{1}{10}$. Then, $\lambda c-\beta h=\frac{1}{20}>0, \beta(\lambda c-\beta h)-a c d=\frac{23}{1000}>0$ and equation (17) is given by

$$
w^{6}-\frac{21}{50} w^{4}+\frac{1731}{5000} w^{2}+\frac{529}{1000000}=0 .
$$

This equation admits two pure imaginary roots given by

$$
\frac{1 / 20 i \sqrt{2} \sqrt{\sqrt[3]{174036+4 \sqrt{5409904729}}\left((174036+4 \sqrt{5409904729})^{2 / 3}-3832-28 \sqrt[3]{174036+4 \sqrt{5409904729}}\right)}}{\sqrt[3]{174036+4 \sqrt{5409904729}}}
$$

and

$$
\frac{-1 / 20 i \sqrt{2} \sqrt{\sqrt[3]{174036+4 \sqrt{5409904729}}\left((174036+4 \sqrt{5409904729})^{2 / 3}-3832-28 \sqrt[3]{174036+4 \sqrt{5409904729}}\right)}}{\sqrt[3]{174036+4 \sqrt{5409904729}}}
$$

Therefore, from Rouché theorem, we cannot conclude anything about the stability of the CTL equilibrium $E_{2}$.

Remark 5 According with different studies, the eclipse phase represented by the time delay $\tau$ can take from 7 to 21 days $[2,3,4,9]$. Based on this, let us assume $\tau=10$ days. In Section 5 we show, numerically, that the infected equilibrium $E_{2}$ is locally asymptotically stable for $\tau=10$ and the parameter values from Table 1 with $\beta=0.5$. This is easy to show analytically: the characteristic equation (16) in this case is given by $q\left(\lambda_{1}\right)=0$ with

$$
q\left(\lambda_{1}\right)=-1 / 4 \mathrm{e}^{-\lambda_{1} \tau} \lambda_{1}-\frac{5}{2} \mathrm{e}^{-\lambda_{1} \tau} \lambda_{1}{ }^{2}+\frac{23}{1000}+\frac{73 \lambda_{1}}{100}+\frac{27 \lambda_{1}{ }^{2}}{10}+\lambda_{1}{ }^{3},
$$

$q(0)=23 / 1000$, and the derivative is always positive for $\lambda_{1} \geq 0$. Therefore, $q\left(\lambda_{1}\right)$ does not have nonnegative real roots. Analogously, we can show that $E_{2}$ is locally asymptotically stable for other positive values of the time delay $\tau$. This will be considered in Section 5. 


\section{Optimal control problem with state and control delays}

It is interesting to introduce drug therapy into the model by assuming that treatment reduces the rate of viral replication, expressed by $(1-u) \beta x y$, where $0 \leq u \leq 1$. Our aim is to find a treatment strategy $u(t)$ that maximizes the number of $C D 4^{+}$ $T$ cells $x$ as well as the number of CTL immune response cells $z$, keeping the cost, measured in terms of chemotherapy strength and a combination of duration and intensity, as low as possible. Due to the importance of the pharmacological delay in the HIV treatment, we consider a discrete time delay in the control variable $u(t)$, denoted by $\xi$, which represents the delay that occurs between the administration of drug and its appearance within cells, due to the time required for drug absorption, distribution, and penetration into the target cells [15]. Precisely, we propose the following control system with discrete time delays in state and control variables:

$$
\left\{\begin{array}{l}
\dot{x}(t)=\lambda-d x(t)-(1-u(t-\xi)) \beta x(t) y(t), \\
\dot{y}(t)=(1-u(t-\xi)) \beta x(t-\tau) y(t-\tau)-a y(t)-p y(t) z(t), \\
\dot{z}(t)=c x(t) y(t) z(t)-h z(t) .
\end{array}\right.
$$

The initial conditions for the state variable $z$ and, due to the delays, initial functions for the state variables $x$ and $y$ and control $u$, are given by

$$
\begin{aligned}
& z(0)=z_{0} \geq 0, \\
& x(t) \equiv x_{0} \geq 0 \text { for }-\tau \leq t \leq 0, \\
& y(t) \equiv y_{0} \geq 0 \text { for }-\tau \leq t \leq 0, \\
& u(t) \equiv u_{0}, \text { where } u_{0} \in[0,1], \text { for }-\xi \leq t<0 .
\end{aligned}
$$

The set of admissible control functions is given by

$$
\Theta=\left\{u(\cdot) \in L^{1}\left(\left[0, t_{f}\right], \mathbb{R}\right) \mid 0 \leq u(t) \leq 1 \forall t \in\left[0, t_{f}\right]\right\}
$$

and the objective functional is

$$
J(u(\cdot))=\int_{0}^{t_{f}}[x(t)+z(t)-u(t)] d t
$$

which measures the concentration of $C D 4^{+} \mathrm{T}$ and $\mathrm{CTL}$ cells and the cost measured in terms of chemotherapy strength and a combination of duration and intensity. The optimal control problem consists in determining a control function $u(\cdot) \in \Theta$ that maximizes the cost functional (20) subject to the control system (18) and initial conditions (19). In [5], the authors consider a different $L^{2}$ cost functional for a non-delayed control system. We claim that our delayed control system (18) describes better the reality. Moreover, as we shall see, the extremals obtained with our $L^{1}$ cost functional (20) are easier to implement from a medical point of view.

We apply the optimality conditions given by the Pontryagin Maximum Principle for multiple delayed optimal control problems of Göllmann and Maurer [6, Theorem 3.1]. For that, we introduce the delayed state variables $\zeta(t)=x(t-\tau), \eta(t)=y(t-\tau)$ and the control variable $v(t)=u(t-\xi)$. Using the adjoint variable $\psi=\left(\lambda_{x}, \lambda_{y}, \lambda_{z}\right) \in \mathbb{R}^{3}$, the Hamiltonian for the cost functional (20) and the control system (18) is given by

$$
H(x, \zeta, y, \eta, z, \psi, u, v)=x+z-u+\lambda_{x}(\lambda-d x-(1-v) \beta x y)+\lambda_{y}((1-v) \beta \zeta \eta-a y-p y z)+\lambda_{z}(c x y z-h z) .
$$

The adjoint equations are given by

$$
\left\{\begin{array}{l}
\dot{\lambda}_{x}(t)=-H_{x}[t]-\chi_{\left[0, t_{f}-\tau\right]} H_{\zeta}[t+\tau] \\
\dot{\lambda}_{y}(t)=-H_{y}[t]-\chi_{\left[0, t_{f}-\tau\right]} H_{\eta}[t+\tau] \\
\dot{\lambda}_{z}(t)=-H_{z}[t]
\end{array}\right.
$$

where the subscripts denote partial derivatives and $\chi_{\left[0, t_{f}-\tau\right]}$ is the characteristic function on the interval $\left[0, t_{f}-\tau\right]($ see $[6])$. Since the terminal state is free, i.e., $\left(x\left(t_{f}\right), y\left(t_{f}\right), z\left(t_{f}\right)\right) \in \mathbb{R}^{3}$, the transversality conditions

$$
\lambda_{x}\left(t_{f}\right)=\lambda_{y}\left(t_{f}\right)=\lambda_{z}\left(t_{f}\right)=0
$$

hold. To characterize the optimal control $u$, we introduce the following switching function:

$$
\begin{aligned}
\phi(t) & =H_{u}[t]+\chi_{\left[0, t_{f}-\xi\right]} H_{v}[t+\xi] \\
& =\left\{\begin{array}{l}
-1+\lambda_{x}(t+\xi) \beta x(t+\xi) y(t+\xi)-\lambda_{y} \beta \zeta(t+\xi) \eta(t+\xi) \quad \text { for } \quad 0 \leq t \leq t_{f}-\xi, \\
-1 \text { for } t_{f}-\xi \leq t \leq t_{f} .
\end{array}\right.
\end{aligned}
$$

The maximality condition of the Pontryagin Maximum Principle [6, Theorem 3.1] gives the control law

$$
u(t)=\left\{\begin{array}{rll}
1 & \text { if } \quad \phi(t)>0, \\
0 & \text { if } \phi(t)<0, \\
\text { singular } & \text { if } \quad \phi_{k}(t)=0 \text { on } I_{s} \subset\left[0, t_{f}\right] .
\end{array}\right.
$$




\section{Numerical simulations}

We begin by showing numerically, in Section 5.1, the local stability of the equilibrium points $E_{0}$ and $E_{2}$ that was proved in Section 3. Then, in Section 5.2, we apply the necessary conditions of optimal control of Section 4 to a situation of fast convergence to the CTL equilibrium $E_{2}$.

\subsection{Stability of the equilibrium points}

Following [5, 18], we consider the parameter values of Table 1.

\begin{tabular}{c|l|r}
\hline Parameter & Description & Value \\
\hline$\lambda$ & source rate of CD4 $+T$ cells & 1 cells/day \\
$d$ & decay rate of CD4 $+T$ cells & 0.1 cells/day \\
$\beta$ & rate CD4 $+T$ cells become infected & $0.00025,0.5]$ cells/day \\
$a$ & death rate infected, not by CTL killing & 0.2 cells/day \\
$p$ & rate at which infected cells are killed by CTLs & $1 /$ day \\
$c$ & immune response activation rate & $0.1 /$ day \\
$h$ & death rate of CTLs & $0.1 /$ day \\
\hline
\end{tabular}

Table 1. Parameter values.

Two different initial conditions for the state variable $z$ and, due to the delays, initial functions for the state variables $x$ and $y$, are considered:

$$
\begin{aligned}
& x(t) \equiv 45, \quad y(t) \equiv 3, \quad-\tau \leq t \leq 0, \\
& z(0)=20,
\end{aligned}
$$

and

$$
\begin{aligned}
& x(t) \equiv 5, \quad y(t) \equiv 1, \quad-\tau \leq t \leq 0, \\
& z(0)=2 .
\end{aligned}
$$

Consider the parameter values of Table 1 and $\beta=0.00025$. For these parameter values, we have $\beta \lambda-d a=-0.0208<0$. Let the time delay $\tau$ be equal to 10 days: $\tau=10$. In Figure 1 we observe the convergence of the variables $x, y, z$ to the steady state $E_{0}=\left(\frac{\lambda}{d}, 0,0\right)=(10,0,0)$.

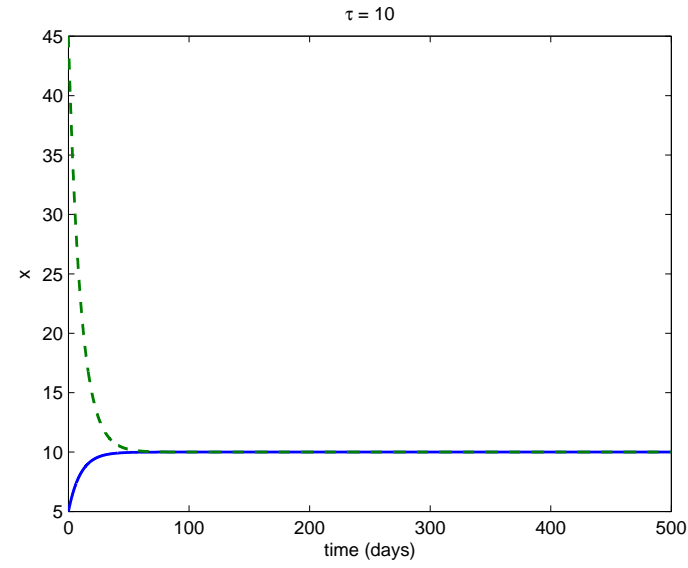

(a) $x(t), t \in[0,500]$

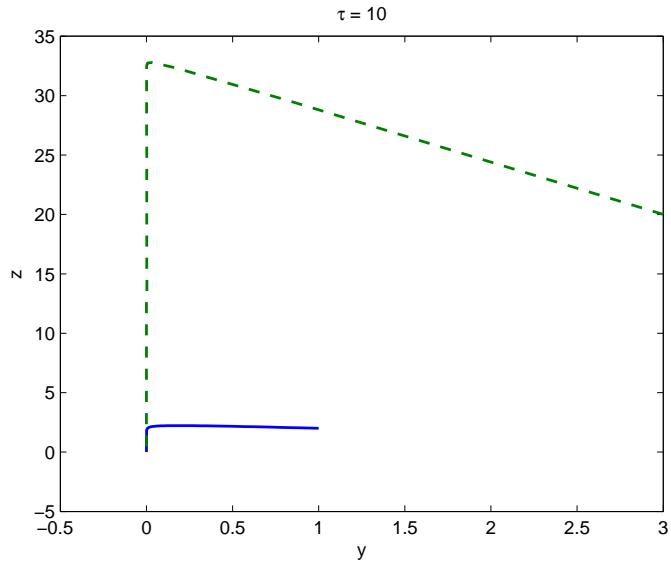

(b) $(y(t), z(t), t \in[0,500]$

Figure 1. Infection-free equilibrium $E_{0}$ for parameter values given in Table $1, \beta=0.00025$ and time delay $\tau=10$ days. The dashed line corresponds to the initial conditions (22) and the continuous line corresponds to the initial conditions (23).

The initial conditions (23) are closer to the infected equilibrium point $E_{2}$ for the parameter values of Table 1 and $\beta=0.5$. For these parameter values, one has $\beta \lambda-d a=0.48>0, \lambda c-\beta h=0.05>0$ and $\beta(\lambda c-\beta h)-a c d=0.023>0$. In Figure 2, 
we observe the convergence of the variables $x, y, z$ to the steady state $E_{2}=\left(\frac{\lambda c-\beta h}{c d}, \frac{d h}{\lambda c-\beta h}, \frac{\beta(\lambda c-\beta h)}{c d p}-\frac{a}{p}\right)=(5,0.2,2.3)$ by considering the initial conditions (22) and (23) and $\tau=10$.

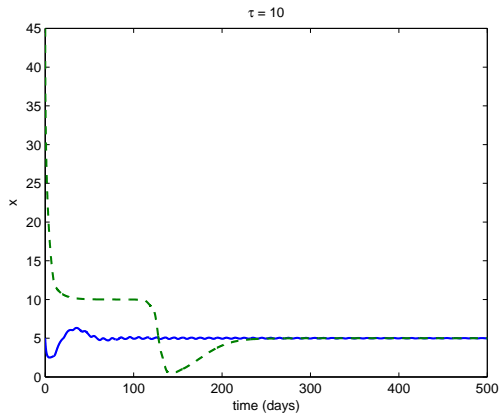

(a) $x(t), t \in[0,500]$

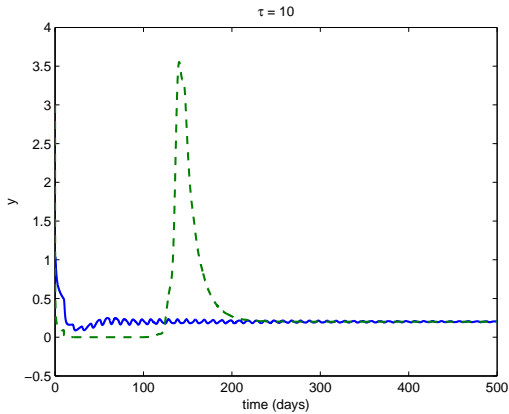

(b) $y(t), t \in[0,500]$

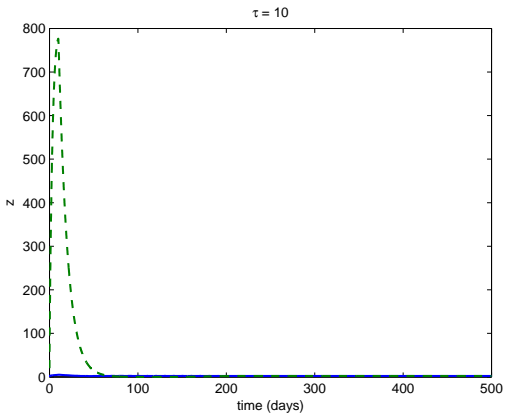

(c) $z(t), t \in[0,500]$

Figure 2. Endemic CTL equilibrium $E_{2}$ for the parameter values given in Table $1, \beta=0.5$ and time delay $\tau=10$. The dashed line corresponds to the initial conditions (22) and the continuous line corresponds to the initial conditions (23).

There are situations where the convergence to stability is much slower. This is illustrated in Figure 3: slower convergence for $\tau=10$ versus faster convergence for $\tau=1$.

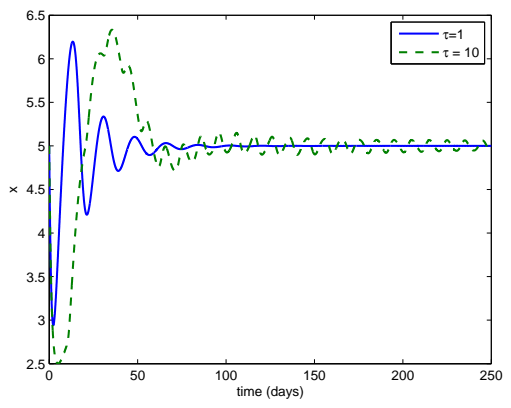

(a) $x(t), t \in[0,250]$

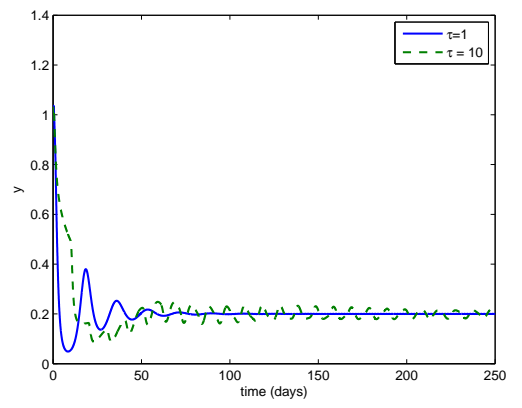

(b) $y(t), t \in[0,250]$

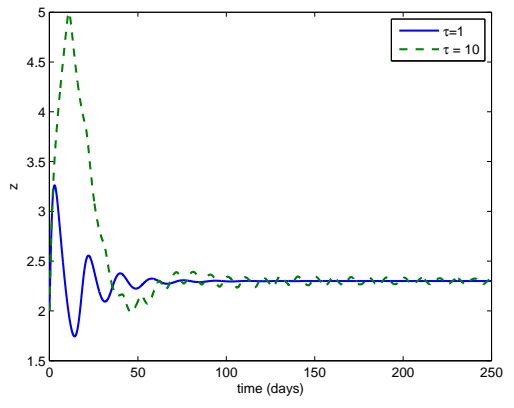

(c) $z(t), t \in[0,250]$

Figure 3. Endemic CTL equilibrium $E_{2}$ for parameter values given in Table $1, \beta=0.5$ and initial conditions (23). The dashed line corresponds to $\tau=10$ and the continuous line corresponds $\tau=1$.

\subsection{Optimal control}

Let us consider the initial conditions (23) and the initial function for the control given by

$$
u(0) \equiv 0, \quad-\xi \leq t<0 .
$$

The extremal for the non-delayed (i.e., $\tau=\xi=0$ ) optimal control problem with a $L^{2}$ functional was investigated in [5]. Figure 4 shows that the extremal control for the $L^{1}$ functional (20) is completely different from the $L^{2}$ case studied in [5]: with $\tau=\xi=0$ and the $L^{1}$ functional (20), the extremal control is bang-bang with several switchings while the $L^{2}$ control extremal [5] is singular after an initial short period of time. We conclude that the $L^{1}$ functional (20) is more suitable, from a medical point of view, because a bang-bang control is much easier to implement than the singular control of [5]. Moreover, we note that in [5] the extremal trajectory $z(t)$ is zero at the final time and the objective is to maximize $x(t)$ and $z(t)$. Our extremal $z(t)$ is always positive and is therefore better than the one of [5]: compare our Figure 4 with Figure 4.1 on page 557 of [5].

Let us now consider an optimal control problem with both incubation and pharmacological time delays. For illustrative purposes, let $\tau=0.5$ and $\xi=0.1$. We see from Figure 5 that the extremal state variables are similar to the ones without delay shown in Figure 4. Importantly, the number of switchings of the extremal control has decreased, which makes it even more simpler to implement in practice. 


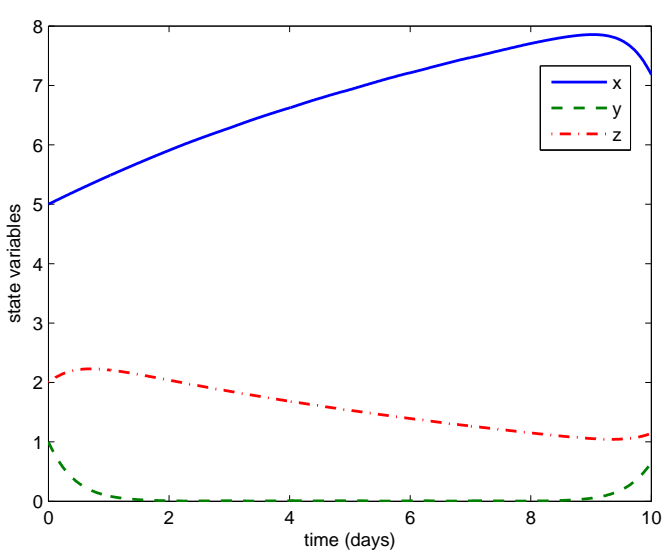

(a) $x(t), y(t)$ and $z(t)$

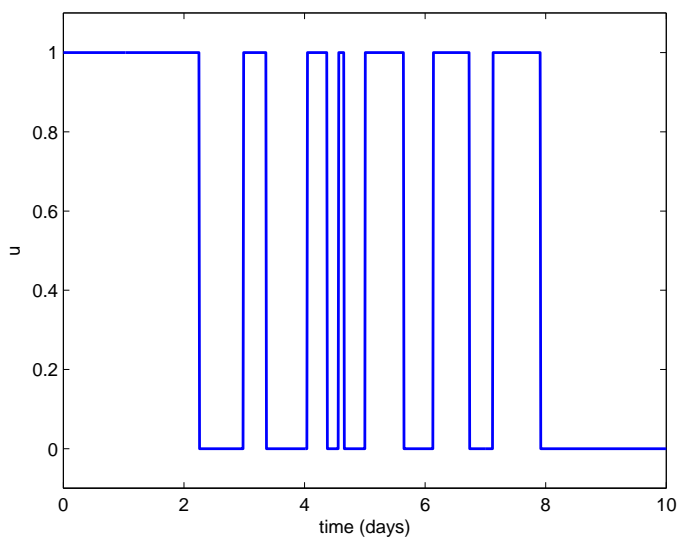

(b) $u(t)(21)$

Figure 4. Extremal solutions of the optimal control problem for $t \in[0,10]$ and $\tau=\xi=0$.

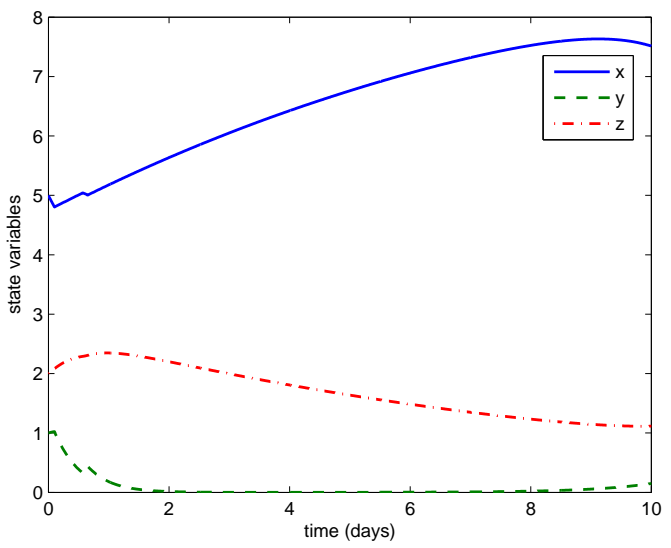

(a) $x(t), y(t)$ and $z(t)$

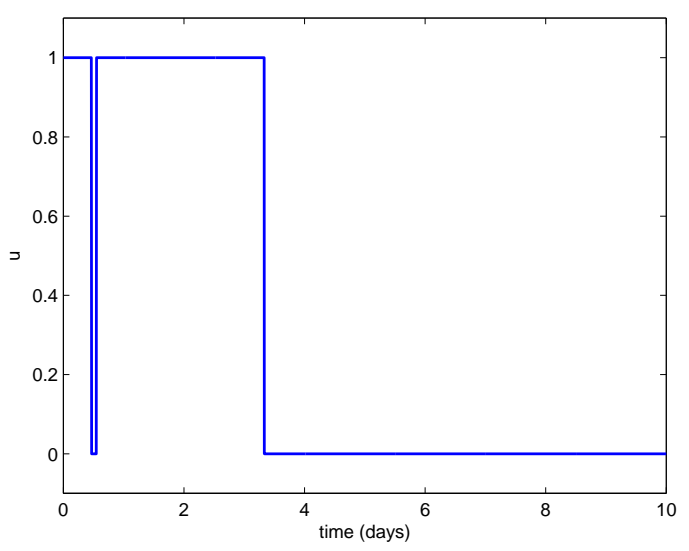

(b) $u(t)(21)$

Figure 5. Extremal solutions of the optimal control problem for $t \in[0,10], \tau=0.5$ and $\xi=0.1$.

\section{Conclusion}

We have proposed a new model for the optimal control of HIV at cell level, which considers not only an intracellular delay (delay $\tau$ in the state variables) but also a pharmacological delay (delay $\xi$ in the control function). Local stability of the equilibria was investigated and the extremal control derived from application of the Pontryagin necessary optimality condition of Göllmann and Maurer [6, Theorem 3.1].

The extremal control for our optimal control problem, with the same values for the parameters as those of [5, 18], is bangbang, that is, it attains alternately the boundary values 0 and 1 . This type of control is easier to implement, from a medical point of view, and leads to better results than the ones previously obtained in [5] for a non-delayed problem with a $L^{2}$ functional.

We offer to the community three open questions: (i) how to prove stability of the CTL equilibrium (7) for an arbitrary $\tau>0$ (see Remark 4); (ii) how to prove sufficient conditions of optimality for our problem with delays in both state and control variables; (iii) how to solve our optimal control problem numerically when one increases $t_{f}, \tau$ and $\xi$.

\section{Acknowledgements}

This research was partially supported by the Portuguese Foundation for Science and Technology (FCT) within projects UID/MAT/04106/2013 (CIDMA) and PTDC/EEI-AUT/2933/2014 (TOCCATTA), co-funded by FEDER funds through COMPETE2020 - Programa Operacional Competitividade e Internacionalização (POCl) and by national funds (FCT). 
Rocha is also supported by the FCT Ph.D. fellowship SFRH/BD/107889/2015; Silva by the FCT post-doc fellowship SFRH/BPD/72061/2010

\section{References}

1. R. A. Arnaout, M. A. Nowak and D. Wodarz, HIV-1 dynamics revisited: biphasic decay by cytotoxic T lymphocyte killing?, Proc. Roy. Soc. Lond. B 265 (2000), 1347-1354.

2. M. P. Busch and G. A. Satten, Time course of viremia and antibody seroconversion following human immunodeficiency virus exposure, Am. J. Med. 102(5B) (1997), 117-126.

3. M. S. Cohen, C. L. Gay, M. P. Busch and F. M. Hecht, The detection of acute HIV infection, Journal of Infectious Diseases 202 (2010), S270-S277.

4. R. W. Coombs, Clinical laboratory diagnosis of HIV-1 and use of viral RNA to monitor infection. In K. K. Holmes (editor), Sexually Transmitted Diseases, New York, McGraw-Hill, 2008.

5. R. Culshaw, S. Ruan and R. Spiteri, Optimal HIV treatment by maximising immune response, J. Math. Biol. 48 (2004), 545-562.

6. L. Göllmann and H. Maurer, Theory and applications of optimal control problems with multiple time-delays, Special Issue on Computational Methods for Optimization and Control, J. Ind. Manag. Optim. 10 (2014), 413-441.

7. J. K. Hale and S. M. V. Lunel, Introduction to Functional Differential Equations, Springer-Verlag, New York, 1993.

8. K. Hattaf and N. Yousfi, Optimal Control of a Delayed HIV Infection Model with Immune Response Using an Efficient Numerical Method, ISRN Biomathematics (2012), 1-7.

9. O. James, M. D. Kahn, D. Bruce and M. D. Walker, Acute Human Immunodeficiency Virus Type 1 infection, N. Engl. J. Med. 339 (1998), 33-39

10. A. Kaddar, A. Abta and H. T. Alaoui, A comparison of delayed SIR and SEIR epidemic models, Nonlinear Anal. Model. Control 16 (2011), no. 2, 181-190

11. Y. Kuang, Delay Differential Equations with Applications in Population Dynamics, Academic Press, San Diego, 1993

12. D. Li and W. Ma, Asymptotic properties of a HIV-1 infection model with time delay, J. Math. Anal. Appl. 335 (2007), no. 1, 683-691.

13. J. E. Mittler, B. Sulzer, A. U. Neumann and A. S. Perelson, Influence of delayed viral production on viral dynamics in HIV-1 infected patients, Math. Biosci. 152 (1998), 143-163.

14. P. W. Nelson, J. D. Murray and A. S. Perelson, A model of HIV-1 pathogenesis that includes an intracellular delay, Math. Biosci. 163 (2000), 201-215.

15. A. S. Perelson, A. U. Neumann, M. Markowitz, J. M. Leonard and D. D. Ho, HIV-1 dynamics in vivo: virion clearance rate, infected cell life-span, and viral generation time, Science, 271 (1996), 1582-1586.

16. C. J. Silva, H. Maurer and D. F. M. Torres, Optimal control of a tuberculosis model with state and control delays, Math. Biosci. Eng. 14 (2017), no. 1 , in press. DOl:10.3934/mbe.2017021 arXiv: 1606.08721

17. C. J. Silva and D. F. M. Torres, A TB-HIV/AIDS coinfection model and optimal control treatment, Discrete Contin. Dyn. Syst. 35 (2015), no. 9, 4639-4663. arXiv:1501.03322

18. D. Wodarz and M. A. Nowak, Specific therapy regimes could lead to long-term immunological control of HIV, Proc. Natl. Acad. Sci. 96 (1999), 14464-14469.

19. H. Zhu and X. Zou, Dynamics of a HIV-1 infection model with cell-mediated immune response and intracellular delay, Discrete Contin. Dyn. Syst. B 12 (2009), 511-524. 